\title{
C-kit Expression Distinguishes Salivary Gland Adenoid Cystic Carcinoma from Polymorphous Low-Grade Adenocarcinoma
}

\author{
Carla R. Penner, D.D.S., Andrew L. Folpe, M.D., Steven D. Budnick, D.D.S. \\ Department of Pathology and Laboratory Medicine, Emory University, Atlanta, Georgia
}

\begin{abstract}
Adenoid cystic carcinoma (ACC) is characterized by persistent, relentless growth and a high rate of eventual metastasis. In contrast, polymorphous low-grade adenocarcinoma (PLGA) has a much lower risk of recurrence and rarely metastasizes. The histologic patterns of these two neoplasms can be similar. Expression of c-kit, a transmembrane receptor tyrosine kinase, has recently been reported to be expressed in ACC but not PLGA. Expression of galectin-3, a nonintegrin $\beta$-galactosidase-binding lectin, has been reported to be significant in PLGA and decreased in ACC.Formalin-fixed paraffinembedded tissue from 9 ACC and 14 PLGA were immunostained for c-kit and galectin-3. Cases were scored as $1+(5-25 \%$ positive $), 2+(26-50 \%$ positive), or $3+(>50 \%$ positive). C-kit was expressed by $100 \%$ of ACC (3+: 7 cases; 2+: 1 case; $1+: 1$ case) and by $57 \%$ of PLGA ( $2+: 2$ cases; $1+: 6$ cases). In all but one ACC, c-kit expression was confined to the inner cell layer. $C$-kit expression was also noted in the intercalated duct epithelium of the salivary glands and the acinar cells of the lacrimal gland. Galectin-3 was expressed in 8 of 9 cases of ACC and 14 of 14 cases of PLGA.The results of this, the first study to compare c-kit and galectin-3 expression in ACC and PLGA, suggest that c-kit expression characterizes ACC, but not PLGA. Galectin-3 immunohistochemistry does not have a role in the differentiation of ACC and PLGA. C-kit immunostaining may be a valuable adjunctive tool for this differential diagnosis, particularly in the setting of a limited biopsy. Our finding of different patterns of c-kit expression in tubular and solid variants of ACC supports the concept of solid variant ACC as a high-
\end{abstract}

Copyright (C) 2002 by The United States and Canadian Academy of Pathology, Inc.

VOL. 15, NO. 7, P. 687, 2002 Printed in the U.S.A.

Date of acceptance: April 3, 2002.

Address reprint requests to: Steven D. Budnick, D.D.S., C-179, Emory University Hospital, Department of Pathology and Laboratory Medicine, 1364 Clifton Road NE, Atlanta, GA 30322; e-mail: sbudnic@emory.edu; fax: 404-712-4780.

DOI: 10.1097/01.MP.0000018973.17736.F8 grade tumor, with progression toward an entirely "inner cell" phenotype.

KEY WORDS: Adenoid cystic carcinoma, C-kit, Galectin, Immunohistochemistry, Polymorphous lowgrade adenocarcinoma.

Mod Pathol 2002;15(7):687-691

The differential diagnosis of polymorphous lowgrade adenocarcinoma (PLGA) and adenoid cystic carcinoma (ACC) remains difficult. Although these neoplasms exhibit similar histologic patterns, their biologic behavior is significantly different. Both ACC and PLGA are believed to originate from the intercalated duct, as both are composed of both luminal epithelial and abluminal myoepithelial cells. ACC is characterized by slow relentless growth, with late metastasis. ACCs typically arise in the parotid and submandibular glands; may exhibit cribiform, tubular, and solid patterns; have hyperchromatic, angulated nuclei; and commonly show prominent perineural invasion (1). In contrast, PLGA is seen almost exclusively in minor salivary glands and is characterized by bland, uniform nuclei with a wide variety of growth patterns, infiltrative growth, and prominent neurotropism. PLGA has a much lower recurrence rate and rarely metastasizes (1-3).

The c-kit proto-oncogene encodes a transmembrane receptor-type tyrosine kinase, which belongs to a family of receptors that includes those for colony-stimulating factor-1 (CSF-1) and plateletderived growth factors (PDGF; 4-6). After binding to the ligand stem cell factor, KIT begins a signal cascade that contributes to the growth and differentiation of multiple hematopoietic lineages (7). C-kit is known to have a significant role in the normal migration and development of germ cells and melanocytes (8).

The C-kit gene product is expressed in several normal cell types including mast cells, interstitial cells of Cajal, melanocytes, and epithelial cells of the breast (9). Alteration in KIT expression is seen in 
a variety of neoplasms, including mastocytosis, gastrointestinal stromal cell tumors (GISTs), and germ cell tumors. C-kit expression has recently been reported in ACC $(8,10)$. In the only previous study of c-kit expression in PLGA, Jeng et al. (8) showed no expression in 4 cases.

Galectins are a family of nonintegrin $\beta$-galactosidase-binding lectins with related amino acid sequences and carbohydrate specificity that have been linked to various physiologic and pathologic processes. One of the best-characterized members of the galectin family is galectin-3. Altered expression of galectins has been associated with neoplastic transformation and progression in several human tumors $(11,12)$. It has been reported that galectin-3 expression is present in PLGA and decreased in ACC (13).

The purpose of this study was to evaluate an immunohistochemical panel consisting of c-kit and galectin-3 in differentiating ACC from PLGA.

\section{MATERIALS AND METHODS}

Fourteen cases of PLGA and 9 cases of ACC were retrieved from the case files of Emory University Hospital, Grady Memorial Hospital, University of Missouri-Kansas City School of Dentistry, and University of Indiana School of Dentistry. The diagnoses were confirmed independently by two oral and maxillofacial pathologists (CP and SB) using accepted criteria (1). Only those cases in which the diagnosis was easily recognizable on hematoxylin and eosin-stained sections were used.

For immunohistochemistry, sections of formalin fixed, paraffin-embedded tissue $(5 \mu \mathrm{m})$ were immu- nostained for c-kit (polyclonal, 1:200, DAKO Corp., Carpinteria, CA) and galectin-3 (A3A12, 1:100, Research Diagnostics Inc., Flanders, NJ). Sections were deparaffinized and rehydrated. Epitope retrieval was performed in citrate buffer ( $\mathrm{pH}$ 6) using an electric pressure cooker, for 5 minutes at $120^{\circ} \mathrm{C}$, with cooling for 10 minutes before immunostaining. All tissues were then exposed to $3 \%$ hydrogen peroxide for 5 minutes, primary antibody for 25 minutes, biotinylated secondary linking antibody for 25 minutes, streptavidin enzyme complex for 25 minutes, diaminobenzidine as chromogen for 5 minutes, and hematoxylin as counterstain for 1 minute. These incubations were performed at room temperature; between incubations, sections were washed with Tris buffered saline buffer.

The immunostaining was scored as negative ( $<5 \%$ of cells positive), $1+(5-25 \%$ cells positive), $2+(26-50 \%$ positive $)$, or $3+(>50 \%$ positive). Only cytoplasmic staining was considered positive for both c-kit and galectin-3.

\section{RESULTS}

Table 1 summarizes the clinicopathological features of the cases studied and the immunohistochemical results. The ACC group, comprised of three men and six women, had a median age of 57 years (range: $42-80$ y). The tumor sites included five from minor salivary glands, specifically lacrimal glands, ethmoid sinus, sphenoid sinus, buccal mucosa, and palate. The major gland tumors involved the parotid (three cases) and sublingual gland (one case). All sections-showed perineural invasion by the tumor cells. All morphologic patterns classically

TABLE 1. Clinicopathological Features and Immunohistochemical Results

\begin{tabular}{|c|c|c|c|c|c|}
\hline Diagnosis & Age (y)/Sex & Location & $2($ Pattern $\lesssim$ & C-kit & Galectin-3 \\
\hline PLGA & $69 / \mathrm{M}$ & Palate & Cribiform/tubular & 1 & 3 \\
\hline PLGA & $70 / \mathrm{M}$ & Maxilla & Cribiform/solid & 1 & 3 \\
\hline PLGA & $71 / \mathrm{F}$ & Palate & Cribiform/tubular & 2 & 3 \\
\hline PLGA & $83 / \mathrm{F}$ & Palate & Cribiform/tubular & 1 & 3 \\
\hline PLGA & $48 / \mathrm{M}$ & Palate & Tubular/cribiform/solid & 2 & 3 \\
\hline PLGA & $67 / \mathrm{F}$ & Palate & Tubular/cribiform/solid & 0 & 3 \\
\hline PLGA & $72 / \mathrm{F}$ & Buccal mucosa & Tubular/solid & 1 & 3 \\
\hline PLGA & $72 / \mathrm{F}$ & Palate & Tubular/cribiform/solid & 0 & 3 \\
\hline PLGA & $26 / M$ & Buccal mucosa & Cribiform/tubular & 0 & 3 \\
\hline PLGA & $40 / \mathrm{M}$ & Retromolar pad & Cribiform/tubular & 0 & 3 \\
\hline PLGA & $61 / \mathrm{M}$ & Palate & Cribiform/tubular & 1 & 3 \\
\hline PLGA & $69 / \mathrm{F}$ & Palate & Cribiform/tubular & 0 & 3 \\
\hline PLGA & $65 / \mathrm{M}$ & Palate & Cribiform/tubular & 1 & 3 \\
\hline PLGA & $43 / \mathrm{F}$ & Buccal mucosa & Tubular & 1 & 3 \\
\hline ACC & $63 / \mathrm{M}$ & Sublingual gland & Cribiform & 3 & 3 \\
\hline ACC & $45 / \mathrm{F}$ & Parotid & Cribiform & 3 & 3 \\
\hline ACC & $66 / \mathrm{F}$ & Sphenoid sinus & Cribiform/tubular & 3 & 0 \\
\hline $\mathrm{ACC}$ & $45 / \mathrm{M}$ & Lacrimal gland & Cribiform/tubular & 3 & 3 \\
\hline ACC & $73 / \mathrm{F}$ & Ethmoid sinus & Cribiform/tubular & 2 & 2 \\
\hline $\mathrm{ACC}$ & $53 / F$ & Parotid & Cribiform/tubular & 3 & 3 \\
\hline ACC & $80 / \mathrm{F}$ & Buccal mucosa & Cribiform/tubular & 3 & 3 \\
\hline ACC & $42 / \mathrm{M}$ & Maxillary sinus & Cribiform/solid & 3 & 3 \\
\hline ACC & $45 / \mathrm{F}$ & Parotid & Cribiform & 1 & 3 \\
\hline
\end{tabular}

PLGA, polymorphous low-grade adenocarcinoma; ACC, adenoid cystic carcinoma. 
described in ACC, including cribiform, tubular, and solid, were represented.

The median age for the PLGA group, consisting of seven men and seven women, was 61 years (range: 26-83 y). All the tumors arose from minor salivary glands in the palate, buccal mucosa, maxillary ridge, and retromolar pad. Twelve of fourteen sections showed perineural invasion with cribiform, tubular, and solid growth patterns.

As shown in Table 1, C-kit was expressed by $100 \%$ of ACCs. Seven cases showed 3+ staining, one case showed $2+$ staining, and one case showed only $1+$ staining. This last case was a recurrence of ACC in which the original tumor showed $3+$ staining. The tubular and cribiform patterns showed c-kit expression in the inner cell layers of the tumor (Fig. 1A, 1B), whereas the solid patterns showed c-kit expression in all cells (Figs. 2A and 2B). C-kit expression was seen in ductal epithelium in benign salivary glands. Galectin-3 was expressed at $3+$ in 8 of 9 (89\%) cases of ACC (Fig. 1C). Galectin-3 expression was also seen in normal salivary gland tissue, including acinar cells, ductal cells, and overlying epithelium.

Expression of c-kit in PLGA was greatly decreased compared with ACC. Six cases were negative for c-kit, six cases showed $1+$ staining, and two cases showed $2+$ staining (Figs. 3A and 3B). Galectin-3 was expressed at $3+$ in 14 of $14(100 \%)$ cases of PLGA (Fig. 3C).

\section{DISCUSSION}

There have been numerous studies attempting to differentiate ACC from PLGA using immunohisto-
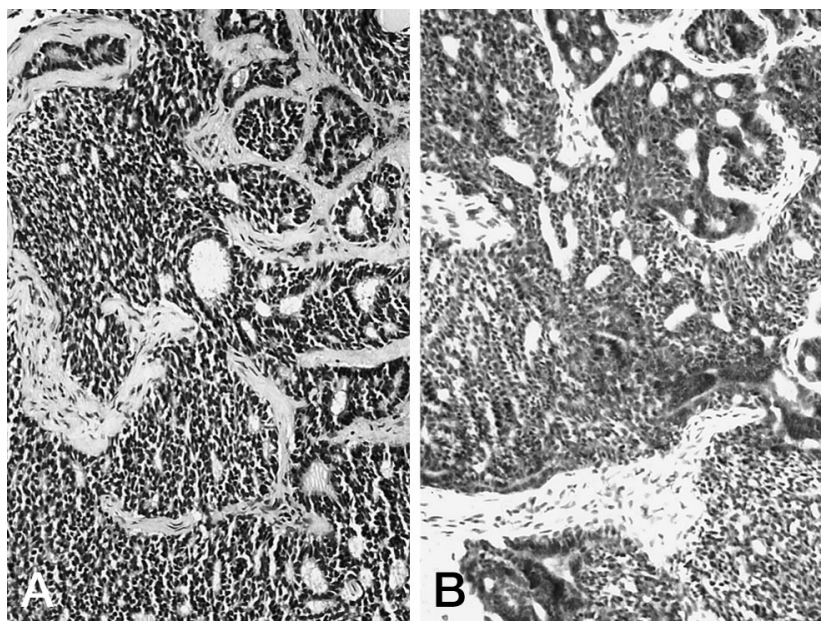

FIGURE 2. Adenoid cystic carcinoma, solid variant (A), showing uniform c-kit expression in all cells (B).

chemistry panels $(2,14-18)$. These various studies have examined the use of antibodies to cytokeratins, vimentin, muscle-specific actin, smoothmuscle myosin heavy chain, and calponin, with variable results $(2,14)$. Although subtle differences may be identified when series of salivary gland tumors are studied, the variability in cellular immunoreactivity limits the effectiveness of previously reported immunohistochemical panels. Although it is beyond the scope of this paper, Ki-67 (MIB1), S-100, and BCL 2 have been reported to be helpful in separating ACC and PLGA on small specimens $(19,20)$.

The strong c-kit expression characteristic of ACC may prove to be a diagnostic tool to differentiate ACC from PLGA. Only 57\% of PLGA were positive
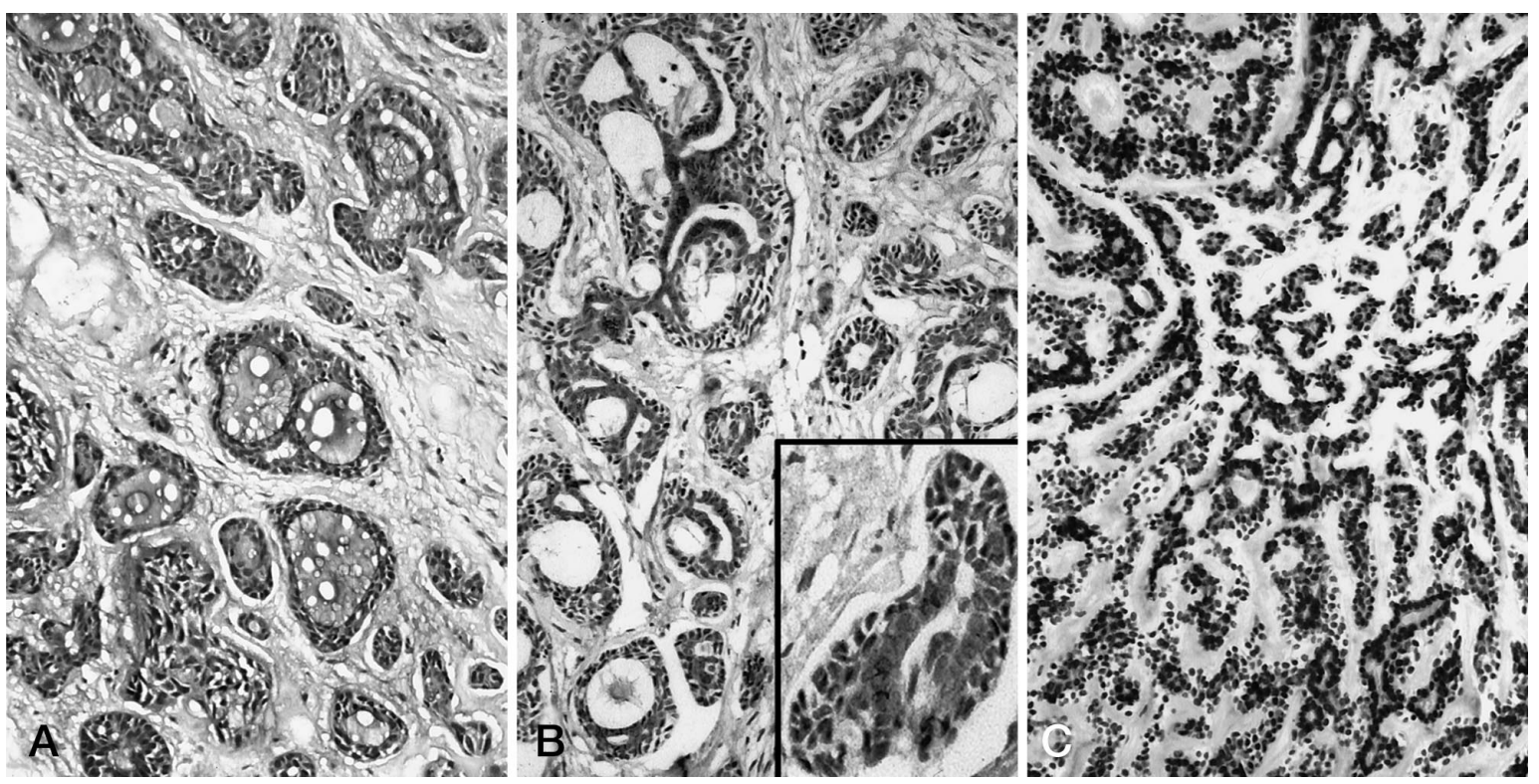

FIGURE 1. Adenoid cystic carcinoma, cribiform pattern (A), showing strong c-kit expression only in the luminal cell population (B). In contrast, all cells showed strong galectin-3 expression $(\mathbf{C})$. 

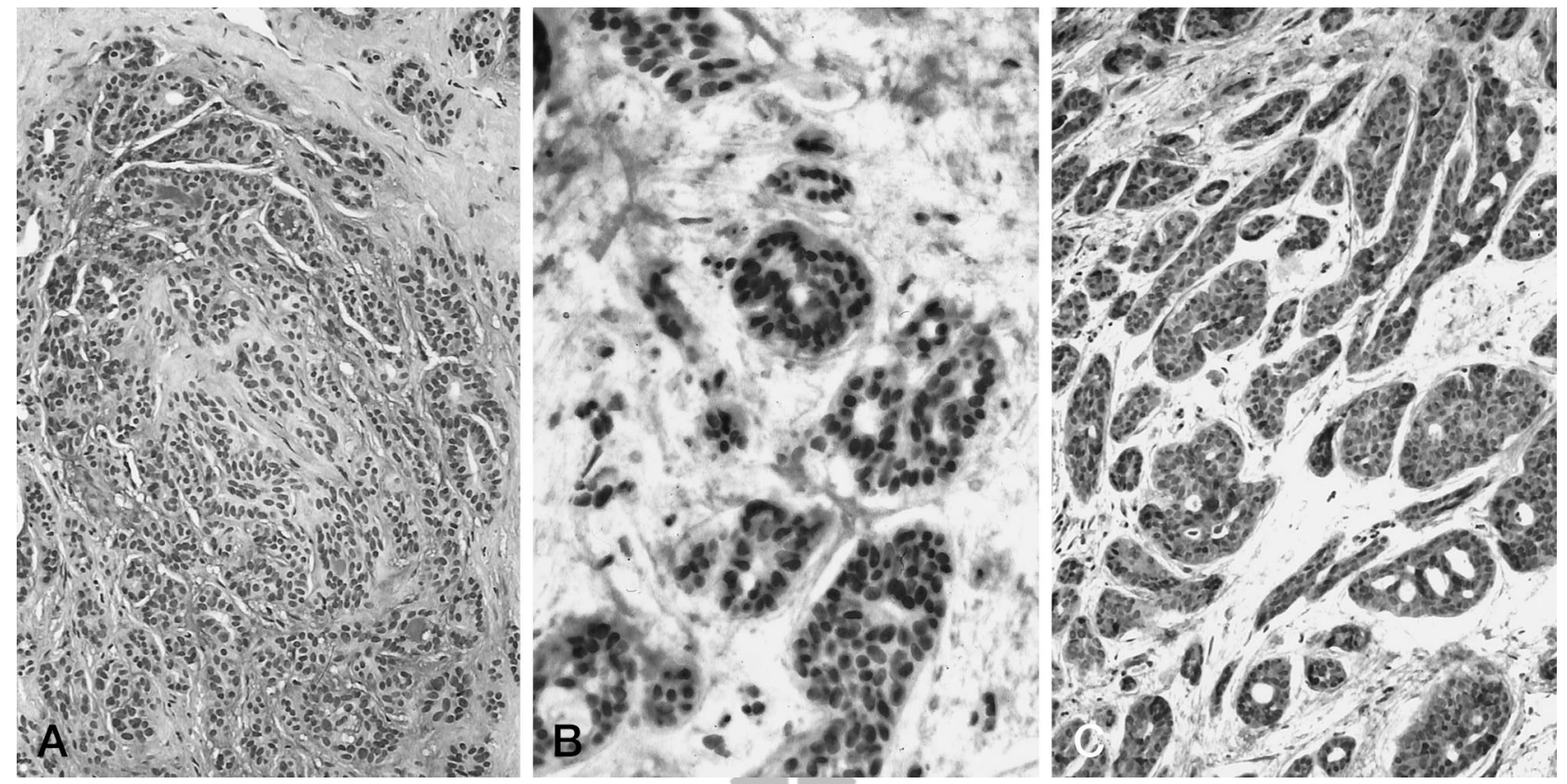

FIGURE 3. Polymorphous low-grade adenocarcinoma (A), showing only very focal expression of c-kit (B). Weak or absent c-kit expression was typical of these tumors. In contrast, galectin-3 was strongly expressed by all cases of PLGA (C).

for c-kit, and these showed only $1+(43 \%)$ or $2+$ (14\%) staining. In contrast, all of ACC showed either $3+$ or $2+$ staining. In histologically ambiguous cases, or in the setting of a limited biopsy, the absence of c-kit expression may serve as additional evidence favoring PLGA over ACC, allowing for the early institution of appropriate therapy.

The pattern of c-kit expression in ACC differs with the histologic subtype. Tubular and/cribiform variants primarily show c-kit expression in the luminal cell layer. This suggests that myoepithelial cells do not express c-kit, as the abluminal cells do not stain (1). Paradoxically, solid variants show expression in all cells, most of which are considered modified myoepithelial cells (1). This difference in the pattern of c-kit expression in tubular and cribiform ACC, as compared with solid variants of ACC, suggests a loss of cellular heterogeneity in solid variants, with differentiation primarily along the lines of the luminal cell layer, and may correlate with the worse clinical course of the solid variant of ACC seen in some studies (1). The role of c-kit overexpression in the aggressive clinical behavior of ACC remains to be elucidated.

Previous studies have shown that galectin-3 was expressed in low-grade salivary gland tumors and reduced in higher grade salivary gland malignancies (13). The exact function of the galectins in human carcinogenesis has yet to be determined. However, in our study, galectin-3, in contrast to c-kit, appears to be ubiquitously expressed by both ACC and PLGA. Galectin-3 was strongly expressed in $89 \%$ of ACC cases and showed in $100 \%$ of PLGAs. Even the solid variants of ACC strongly stained with galectin-3, in contrast to previous findings of decreased expression in high-grade tumors. There does not appear to be a role for galectin-3 immunohistochemistry in the differential diagnosis of ACC and PLGA.

In summary, we believe our study supports a role for c-kit immunohistochemistry in the differential diagnosis of ACC and PLGA. However, we were only able to study a relatively small number of cases, and additional study will be necessary to draw firm conclusions about the utility of c-kit in the differential diagnosis of salivary gland tumors. The uniform strong expression of c-kit seen in this and previous studies of ACC suggests a potential role for c-kit inhibitors such as STI-571 in the treatment of patients with advanced ACC.

\section{0)}

\section{REFERENCES}

1. Ellis GL, Auclair PL. Tumors of the salivary glands. In: Rosai J, editor. Atlas of tumor pathology. Fascicle 17. Washington DC: Armed Forces Institute of Pathology; 1995. p. 203-28.

2. Castle JT, Thompson LDR, Frommelt RA, Wenig BM, Kessler HP. Polymorphous low grade adenocarcinoma, a clinicopathologic study of 164 cases. Cancer. 1999;6(2):207-19.

3. Evans HL, Luna MA. Polymorphous low-grade adenocarcinoma: a study of 40 cases with long-term follow up and an evaluation of the importance of papillary areas. Am J Surg Pathol 2000;4(10):1319-28.

4. Vandenbark GR, deCastro CM, Taylor H, Dew-Knight S, Kaufman RE. Cloning and structural analysis of the human c-kit gene. Oncogene 1992;(7):1259-66.

5. Vliagoftis H, Worobec AS, Metcalfe DD. The protooncogene c-kit and c-kit ligand in human disease. J Allergy Clin Immunol 1997;100(4):435-40.

6. Yarden Y, Kuang WS, Yang-Feng T, Coussens L, Munemitsu $\mathrm{S}$, Dull TJ, et al. Human proto-oncogene c-kit: a new cell 
surface receptor tyrosine kinase for an unidentified ligand. EMBO J 1987;6(11):3341-51.

7. Funasaka Y, Boulton T, Cobb M, Yarden Y, Fan B, Lyman SD, et al. C-kit-kinase induces a cascade of protein tyrosine phosphorylation in normal human melanocytes in response to mast cell growth factor and stimulates mitogen-activated protein kinase but is down-regulated in melanomas. Mol Biol Cell 1992;3(2):197-209.

8. Jeng YM, Lin CY, Hsu HC. Expression of the c-kit protein is associated with certain subtypes of salivary gland carcinoma. Cancer Lett 2000;154(1):107-11.

9. Lammie A, Drobnjak M, Gerald W, Saad A, Cote R, CordonCardo C. Expression of c-kit and kit ligand proteins in normal human tissue. J Histochem Cytochem 1994;42(11):1417-25.

10. Holst VA, Marshall CE, Moskaluk CA, Frierson HF. KIT protein expression and analysis of c-kit gene mutation in adenoid cystic carcinoma. Mod Pathol 1999;12(10):956-60.

11. Raz A, Meromsky L, Zwibel I, Lotan R. Transformationrelated changes in the expression of endogenous cell lectins. Int J Cancer 1987;39(3):353-60.

12. Raz A, Lotan R. Endogenous galactosidase-binding lectins: a new class of functional tumor cell surface molecules related to metastasis. Cancer Metastasis Rev 1987;6(3):433-52.

13. Xu XC, Gallego JJS, Lotan R, El-Naggar AK. Differential expression of galectin-1 and galectin-3 in benign and malignant salivary gland neoplasms. Int J Oncol 2000;17:271-6.

14. Cavalcanti de Araújo V, Orsini Machado de Sousa S, Carvalho YR, Soares de Araújo N. Application of immunohisto- chemistry to the diagnosis of salivary gland tumors. Appl Immunohistochem Mol Morphol 2000;8(3):195-202.

15. Prasad AR, Savera AT, Gown AM, Zarbo RJ. The myoepithelial immunophenotype in 135 benign and malignant salivary gland tumors other than pleomorphic adenoma. Arch Pathol Lab Med 1999;123:801-6.

16. Nordkvist A, Röijer E, Bang G, Gustafsson H, Behrendt M, Ryd W, et al. Expression and mutation patterns of p53 in benign and malignant salivary gland tumors. Int $\mathrm{J}$ Oncol 2000;16:477-83.

17. Huang JW, Ming Z, Shrestha P, Mori M, Ilg E, Schafer BW, et al. Immunohistochemical evaluation of the $\mathrm{CA}(2+)$-binding S-100 proteins S100A1, S100A2, S-100A4, S-100A6 and S-100B in salivary gland tumors. J Oral Pathol Med 1996; 25(10):547-55.

18. Xing R, Regezi JA, Stern M, Shuster S, Stern R. Hyaluronan and CD44 expression in minor salivary gland tumors. Oral Dis 1998;4(4):241-7.

19. Vargas H, Sudilovsky D, Kaplan MJ, Regezi JA, Weidner N. Mixed tumor, polymorphous low-grade adenocarcinoma and adenoid cystic carcinoma of the salivary gland: pathogenic implications and differential diagnosis by Ki-67 (MIB1), BCL2, and S-100 immunohistochemistry. Appl Immunohistochem 1997;5(1):8-16.

20. Skalova A, Simpson RH, Lehtonen H, Leivo I. Assessment of proliferative activity using the MIB1 antibody help to distinguish polymorphous low grade adenocarcinoma from adenoid cystic carcinoma of salivary glands. Pathol Res Pract 1997;193(10):695-703.

\section{Book Review}

\section{Byard RW and Krous HF, editors: Sudden Infant Death Syndrome: Problems, Progress \& Possibilities, 256 pp, Adelaide, Edward Ar- nold, 2001 (\$78.50).}

This is a unique overview of the state of current knowledge regarding Sudden Infant Death Syndrome (SIDS) explored from a range of perspectives. Contributors include basic scientists, forensic pathologists, neuropathologists, microbiologists, clinicians, epidemiologists, and sociologists. Even the personal perspective of a mother who lost her child to SIDS is included. The many perspectives represented reflect the swirl of debate that surrounds this politically charged topic. Interestingly, one of the most hotly debated facets of SIDS is its very definition. In the preface, Dr. J. Bruce Beckwith, who introduced the term SIDS in 1969, argues that two definitions should exist: a rigorous one for scientific publications and a more inclusive one for the purposes of qualification for family support services. Theories of causality abound in the SIDS research community, but/it has been reliably shown that since the introduction of public health campaigns urging parents not to position sleeping infants in the prone position, SIDS rates have declined. But the question remains, as raised by Dr. Susan Beal in her chapter reviewing theories of causality, "Why can some, but not all, infants cope with the prone position? How do we identify such infants?" I highly recommend this review of the current state of SIDS research and diagnosis. Forensic pathologists will find this book of practical use (particularly Dr. Randy Hanzlick's excellent chapter on death scene investigation), while researchers will find it a useful compendium of current opinion in the field.
Brian E. Moore
University of Massachusetts Memorial Medical Center
Worcester, Massachusetts 